\title{
Article \\ Combination of Histone Deacetylase Inhibitor Panobinostat (LBH589) with $\beta$-Catenin Inhibitor Tegavivint (BC2059) Exerts Significant Anti-Myeloma Activity Both In Vitro and In Vivo
}

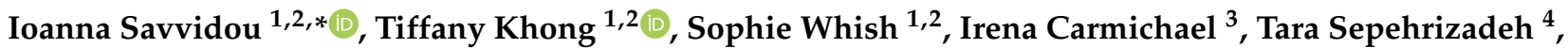 \\ Sridurga Mithraprabhu $\left.{ }^{1,2}{ }^{(}\right)$, Stephen K. Horrigan ${ }^{5}$, Michael de Veer ${ }^{4}$ and Andrew Spencer ${ }^{1,2,6, *}$
}

check for updates

Citation: Savvidou, I.; Khong, T.; Whish, S.; Carmichael, I.; Sepehrizadeh, T.; Mithraprabhu, S.; Horrigan, S.K.; de Veer, M.; Spencer, A. Combination of Histone Deacetylase Inhibitor Panobinostat (LBH589) with $\beta$-Catenin Inhibitor Tegavivint (BC2059) Exerts

Significant Anti-Myeloma Activity Both In Vitro and In Vivo. Cancers 2022, 14, 840. https://doi.org/ 10.3390 /cancers 14030840 Academic Editor: Katja Weisel

Received: 11 January 2022 Accepted: 4 February 2022 Published: 8 February 2022

Publisher's Note: MDPI stays neutral with regard to jurisdictional claims in published maps and institutional affiliations.

Copyright: (C) 2022 by the authors. Licensee MDPI, Basel, Switzerland. This article is an open access article distributed under the terms and conditions of the Creative Commons Attribution (CC BY) license (https:// creativecommons.org/licenses/by/ $4.0 /)$.
1 Myeloma Research Group, Australian Centre for Blood Diseases, Central Clinical School, Faculty of Medicine, Nursing and Health Sciences, Monash University, Melbourne, VIC 3004, Australia; tiffany.khong@monash.edu (T.K.); swhish88@gmail.com (S.W.); durga.mithraprabhu@monash.edu (S.M.)

2 Malignant Haematology and Stem Cell Transplantation, Department of Haematology, Alfred Hospital, Melbourne, VIC 3004, Australia

3 Monash Micro Imaging-Alfred Research Alliance, Faculty of Medicine, Nursing and Health Sciences, Monash University, Melbourne, VIC 3004, Australia; iska.carmichael@monash.edu

4 Monash Biomedical Imaging, Monash Technology Research Platforms, Monash University, Melbourne, VIC 3004, Australia; tara.sepehrizadeh@monash.edu (T.S.); michael.deveer@monash.edu (M.d.V.)

5 Iterion Therapeutics, 2450 Holcombe Blvd, Houston, TX 77021, USA; stephen@iteriontx.com

6 Department of Clinical Hematology, Monash University, Melbourne, VIC 3004, Australia

* Correspondence: ioanna.savvidou@monash.edu (I.S.); andrew.spencer@monash.edu (A.S.); Tel.: +61-3-9903-0602 (I.S.); +61-3-9076-3393 (A.S.)

Simple Summary: Multiple myeloma remains an incurable malignancy with the majority of patients succumbing to the disease after receiving several lines of treatment, while acquiring drug resistance. Although panobinostat, a histone deacetylase inhibitor, has long been approved for the treatment of patients with relapsed/refractory myeloma it has not yet been incorporated into everyday practice. In this study we showed a significant synergistic anti-myeloma effect of low dose panobinostat and $\beta$-catenin inhibitor Tegavivint both in vitro and in vivo, with a favourable toxicity profile. This combination could significantly and safely benefit myeloma patients that have exhausted mainstream therapeutic modalities due to acquisition of drug resistance in the future.

\begin{abstract}
Over the last three decades changes in the treatment paradigm for newly diagnosed multiple myeloma (MM) have led to a significant increase in overall survival. Despite this, the majority of patients relapse after one or more lines of treatment while acquiring resistance to available therapies. Panobinostat, a pan-histone deacetylase inhibitor, was approved by the FDA in 2015 for patients with relapsed MM but how to incorporate panobinostat most effectively into everyday practice remains unclear. Dysregulation of the Wnt canonical pathway, and its key mediator $\beta$-catenin, has been shown to be important for the evolution of MM and the acquisition of drug resistance, making it a potentially attractive therapeutic target. Despite concerns regarding the safety of Wnt pathway inhibitors, we have recently shown that the $\beta$-catenin inhibitor Tegavivint is deliverable and effective in in vivo models of MM. In this study we show that the combination of low concentrations of panobinostat and Tegavivint have significant in vitro and in vivo anti-MM effects including in the context of proteasome inhibitor resistance, by targeting both aerobic glycolysis and mitochondrial respiration and the down-regulation of down-stream $\beta$-catenin targets including myc, cyclinD1, and cyclinD2. The significant anti-MM effect of this novel combination warrants further evaluation for the treatment of MM patients with relapsed and/or refractory MM.
\end{abstract}

Keywords: multiple myeloma; drug resistance; histone deacetylase inhibition; $\beta$-catenin inhibition; combination treatment; in vivo drug synergism 


\section{Introduction}

Multiple Myeloma (MM) is the second most common haematologic malignancy with 176,404 new cases and 117,077 deaths registered worldwide in 2020 [1]. It arises from the malignant transformation of antibody producing plasma cells residing in the bone marrow and is preceded by the premalignant condition MGUS (Monoclonal Gammopathy of Undetermined Significance). While the treatment landscape for MM has changed significantly over the past 30 years with the introduction of proteasome inhibitors (bortezomib, carfilzomib, ixazomib), immunomodulatory drugs (IMiDs) (thalidomide, lenalidomide, pomalidomide) and monoclonal antibodies (daratumumab and elotuzumab), resulting in improved overall survival (OS) [2] most patients eventually relapse, with the duration and depth of response decreasing with each line of therapy [3].

Panobinostat (LBH589) is an oral pan-histone deacetylase (HDAC) inhibitor that, based on the results of the PANORAMA 1 trial [4], was approved by the FDA in 2015 for the treatment of patients with relapsed and/or refractory MM (RRMM) who had received two or more prior lines of therapy, including bortezomib and an IMiD. More recently, the results of the PANORAMA 3 trial exploring alternative dosing and scheduling of panobinostat in combination with sub-cutaneous bortezomib have confirmed both a high level of efficacy and the deliverability of this combination in RRMM [5]. This confirmation provides a rationale for the further evaluation of panobinostat as a novel therapeutic modality in treatment combinations with alternative mechanisms of action to address the ongoing unmet need for more effective therapies for RRMM [6].

The role in tumorigenesis of dysregulation of canonical Wnt signalling and its key player $\beta$-catenin have long been recognized [7]. In relapsed MM, studies have confirmed aberrant activation of the Wnt canonical pathway in malignant plasma cells due to multiple mechanisms [8] and that this activation plays an important role in proliferation, survival, and drug resistance, so identifying the Wnt canonical pathway as a potentially attractive therapeutic target [9]. Tegavivint (BC2059), has been shown to exert potent cytotoxic effects against solid [10] and haematologic malignancies [11,12] including MM [9], with a predicted to be favourable toxicity profile and is currently in early phase clinical development (NCT03459469, NCT04851119, NCT04874480, NCT04780568). It exerts its activity by disrupting the binding of $\beta$-catenin to Transducin $\beta$-like protein 1 (TBL1) and its related protein (TBLR1), which under Wnt activation recognize and preferentially bind to hypoacetylated histones, thus localizing $\beta$-catenin to the Wnt target genes promoter [13,14].

In the present study we show that low concentrations of Tegavivint are able to potentiate the anti-MM effect of panobinostat in vitro, ex vivo, and in vivo including in both panobinostat and bortezomib resistant MM cells, thus providing the rational for the further evaluation of this potential novel therapeutic combination in RRMM.

\section{Materials and Methods}

\subsection{Primary Samples Ex Vivo Treatment}

Primary multiple myeloma samples were obtained from RRMM patients, following written informed consent with approval from the Alfred Hospital Research and Ethics Committee. Bone marrow mononuclear cells (BMMCs) were isolated with Ficoll-Paque Plus (Amersham Biosciences, Uppsala, Sweeden), washed and processed as previously described [9]. For further details see Supplementary Materials and Methods.

\subsection{Myeloma Cell Lines}

MM1R, NCIH929 and U266 Human Myeloma Cell Lines (HMCL) and HS5 (human fibroblasts) were obtained from the ATCC. OCIMy1, and IL6 dependent ANBL6 and XG1 were kind gifts from Frits Van Rhee (Winthrop P. Rockefeller Cancer Institute, Little Rock, AR, USA), whereas KMS34 was a kind gift from Kawasaki Medical School (Kurashiki, Japan) in 2008. HMCLs and HS5 cells were grown and treated at density $2.0 \times 10^{5}$ cells $/ \mathrm{mL}$ in RPMI1640 media (Gibco, Invitrogen, Waltham, MA, USA) supplemented with $10 \%$ heatinactivated FBS (Lonza, Basel, Switzerland) and $2 \mathrm{mmol} / \mathrm{L}$ L-glutamine (Gibco, Invitrogen, 
Waltham, MA USA). $5 \mathrm{ng} / \mathrm{mL}$ of human IL6 (ProSpec-Tany TechnoGene, Ness-Ziona, Israel) was added in the medium for IL6 dependent cell lines ANBL6 and XG1.

\subsection{Confocal Microscopy}

OCIMy1 and U266 cells were treated with Tegavivint $100 \mathrm{nmol} / \mathrm{L}$, panobinostat $10 \mathrm{nmol} / \mathrm{L}$, Tegavivint and panobinostat or vehicle (DMSO) for $20 \mathrm{~h}$ and stained with TMRE (a fluorescent dye that is readily sequestered by active mitochondria thus acting as a marker of mitochondrial fitness) at $100 \mathrm{nmol} / \mathrm{L}$ (Sigma-Aldrich, Merck Pty. Ltd., Bayswater, Australia), Mitotracker Green FM (a green fluorescent mitochondrial stain which localizes to mitochondria regardless of mitochondrial membrane potential) at $200 \mathrm{nmol} / \mathrm{L}$ (ThermoFisher Scientific, Waltham, MA, USA) and Hoechst stain (ThermoFisher Scientific, Waltham, MA, USA) according to the manufacturer's instructions. Carbonyl cyanide 4-(trifluoromethoxy)phenylhydrazone (FCCP) (a potent uncoupler of mitochondrial oxidative phosphorylation which disrupts ATP synthesis by transporting protons across the mitochondrial inner membrane) $1 \mu \mathrm{mol} / \mathrm{L}$ was used as a positive control. Fluorescence images were obtained by using an inverted Nikon A1r confocal microscope (Tokyo, Japan) equipped with a motorized piezo stage Galvano scanner and perfect focus system, using Apo LWD 40x WI $\lambda$ S DIC N2 (numerical aperture 1.15; Nikon, Tokyo, Japan). For further details see Supplementary Materials and Methods.

\subsection{XF Mito Stress Analysis}

OCIMy1, $2 \times 10^{5}$ cells $/ \mathrm{mL}$, and U266 were plated and treated with DMSO, Tegavivint, panobinostat or their combination. At $18 \mathrm{~h}$, cells were counted, centrifuged, and resuspended in the base medium containing $2 \mathrm{mM}$ L-glutamine, $1 \mathrm{mM}$ sodium pyruvate, and $10 \mathrm{mM}$ glucose (Sigma-Aldrich, Merck Pty. Ltd., Bayswater, Australia) and seeded in a 96 well XF Cell Culture Microplate previously treated with Cell-Tak (Corning, Glendale, AZ, USA) according to the manufacturer's instructions, at $5 \times 10^{4}$ cells per well. Drugs were added accordingly. The oxygen consumption rate was measured using a XFe96 extracellular flux analyser (Agilent Technologies, Santa Clara, CA, USA) with sequential injection of $1 \mu \mathrm{mol} / \mathrm{L}$ oligomycin A (O), $1 \mu \mathrm{mol} / \mathrm{L} \mathrm{FCCP,} \mathrm{and} 1 \mu \mathrm{mol} / \mathrm{L}$ rotenone/antimycin A (R/A) (Sigma-Aldrich, Merck Pty. Ltd., Bayswater, Australia).

\subsection{In Vivo Studies}

Approval for the murine studies was obtained from the Animal Ethics Committee of the Alfred Hospital, Melbourne, Australia (E/1376/2031/M). Adult age-matched CgPrkdcscid Il2rgtm1Wjl/SzJ mice (The Jackson Laboratory, Ellsworth, ME, USA) were injected (intravenously) with $1 \times 10^{6} \mathrm{U} 266 \mathrm{HMCL}$, carrying the FUL2-TG vector (a generous gift from Marco Herold, WEHI, Melbourne, Australia; with luciferase 2 and GFP under the constitutively active ubiquitin and IRES promoter, respectively). Treatment was initiated at day 10 upon confirmation of established measurable disease by bioluminescence at day 7. Control mice $(n=5)$ received vehicle $(17 \%$ Solutol HS 15 [Sigma-Aldrich, Merck Pty. Ltd., Bayswater, Australia] and normal saline), whereas treated mice received $30 \mathrm{mg} / \mathrm{kg}$ Tegavivint $(n=5)$ or $10 \mathrm{mg} / \mathrm{kg}$ panobinostat $(n=5)$ or their combination $(n=5)$ twice weekly intraperitoneally for 3 consecutive weeks for each of two 28 days cycles. Tumour burden was monitored on a weekly basis by in vivo imaging until the first mice reached experimental endpoint. Peripheral blood counts were sequentially monitored throughout the course of the experiment (Hemavet, Drew Scientific, Inc., Miami Lakes, FL, USA). Upon reaching the experimental endpoints (hind limb paralysis, $>20 \%$ weight loss) the mice were humanely euthanized and tissues (spine and colon) were collected. Tissues were formalin fixed and embedded in paraffin, sectioned, and stained with H\&E. Images were taken with an Olympus BX51 microscope.2.6. Micro-CT Studies

Images of the 5 th lumbar vertebrae from the mice were acquired at the time of euthanasia using the Siemens Inveon PET/SPECT/CT scanner. The scans were performed using the computer tomography (CT) modality only, and the settings were as follows: voltage 
of $80 \mathrm{kV}$, current of $500 \mathrm{uA}, 360$ projections in step and shoot mode, $9.9 \mu \mathrm{m}$ resolution reconstruction with a murine beam hardening algorithm applied. All reconstructions of the images were created with a reference HU factor for each sample ensuring the data was consistent. For $\mu \mathrm{CT}$ analysis see Supplementary Materials and Methods.

\section{Results}

3.1. Low Dose Combinations of Tegavivint and Panobinostat Exert Synergistic Anti-MM Effects In Vitro and Ex Vivo

HMCL have variable sensitivity to HDACi, including panobinostat, with the $t(11 ; 14)$ positive HMCL U266 being reported as highly resistant to the latter [15]. We determined the effect of panobinostat on the viability of seven genetically heterogeneous HMCL, including U266 and IL6 dependent ANBL6 and XG1, at 48 h (Figure 1A, left panel). Addition of IL6 did not influence the resistance of HMCL to panobinostat. U266 proved to be the most resistant cell line, with an $\mathrm{LD}_{50}=81.3 \mathrm{nmol} / \mathrm{L}$ (Figure 1A, right panel), whereas OCIMy1 [t $(14 ; 16)$ positive] and NCIH929 $[\mathrm{t}(4 ; 14)$ positive] showed intermediate sensitivities with $\mathrm{LD}_{50}$ of 31.9 and $41.8 \mathrm{nmol} / \mathrm{L}$, respectively (Supplementary Figure S1A). All three HMCL have been previously shown to have intermediate sensitivity to Tegavivint, with LD $_{50}$ at $24 \mathrm{~h}$ around $200 \mathrm{nmol} / \mathrm{L}$ [9]. Their dose response to Tegavivint at $48 \mathrm{~h}$ was further validated (Supplementary Figure S1B) and combination treatment with low concentrations of panobinostat $(\leq 10 \mathrm{nmol} / \mathrm{L})$ and Tegavivint $(\leq 150 \mathrm{nmol} / \mathrm{L})$ were evaluated at $48 \mathrm{~h}$. In all three HMCL the combination proved to be highly synergistic, particularly U266 which exhibited the highest synergy score [16] (Bliss synergy score U266 = 26.4, NCIH929 = 13.2, OCIMy1 = 11.4) (Figure 1B). In contrast panobinostat or Tegavivint alone at the same doses did not affect the viability of non-malignant HS5 cells (Figure 1C, left panel). In accordance, combination of the two drugs in HS5 did not induce significant cell death (max relative cell death: $8.28 \%$ with panobinostat $20 \mathrm{nmol} / \mathrm{L}$ and Tegavivint $150 \mathrm{nmol} / \mathrm{L}$ ) (Figure 1C, right panel).

The effect of the combination on ex vivo primary MM cells was subsequently evaluated. Primary MM tumour cells from two patients with RRMM were treated for $72 \mathrm{~h}$, after which the CD38+ CD45- MM cell population was assessed for apoptosis by flow cytometry (Figure 1D). In accordance with the in vitro results the combination of Tegavivint with panobinostat proved to be synergistic with calculated Bliss synergy scores 27.5 and 8.42 respectively (Supplementary Figure S2A). In contrast, the combination had no synergistic pro-apoptotic effect to the non-myeloma stromal cells, with calculated Bliss synergy scores -0.59 and -2.66 respectively (Supplementary Figure S2B). 
A.
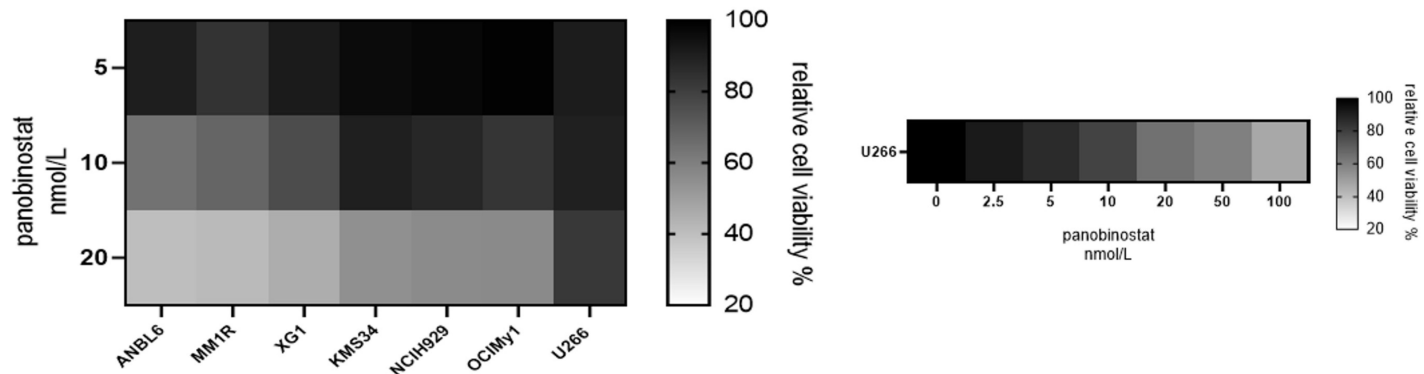

$\mathrm{t}(14 ; 16) \quad \mathrm{t}(14 ; 16) \mathrm{t}(11 ; 14) \quad \mathrm{t}(4 ; 14) \quad \mathrm{t}(4 ; 14) \mathrm{t}(14 ; 16) \mathrm{t}(11 ; 14)$

L6 dependency + + + - -

B.

$\mathrm{NClH} 929$

Bliss synergy score: 13.173

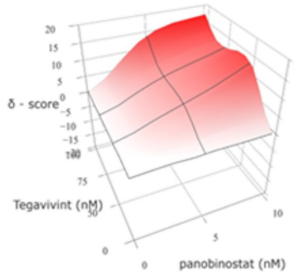

C.
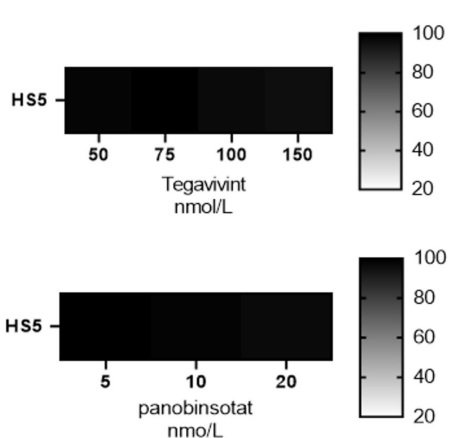

OCIMy1

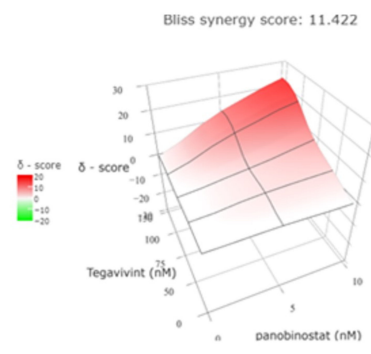

HS5

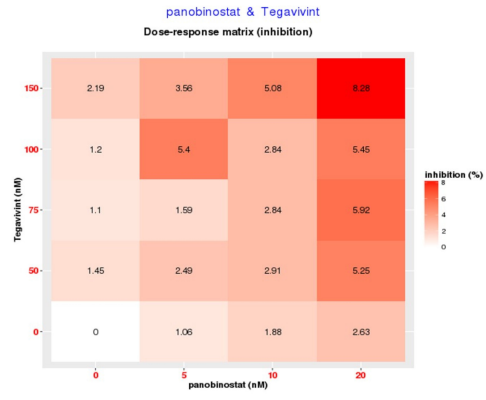

D.
U266
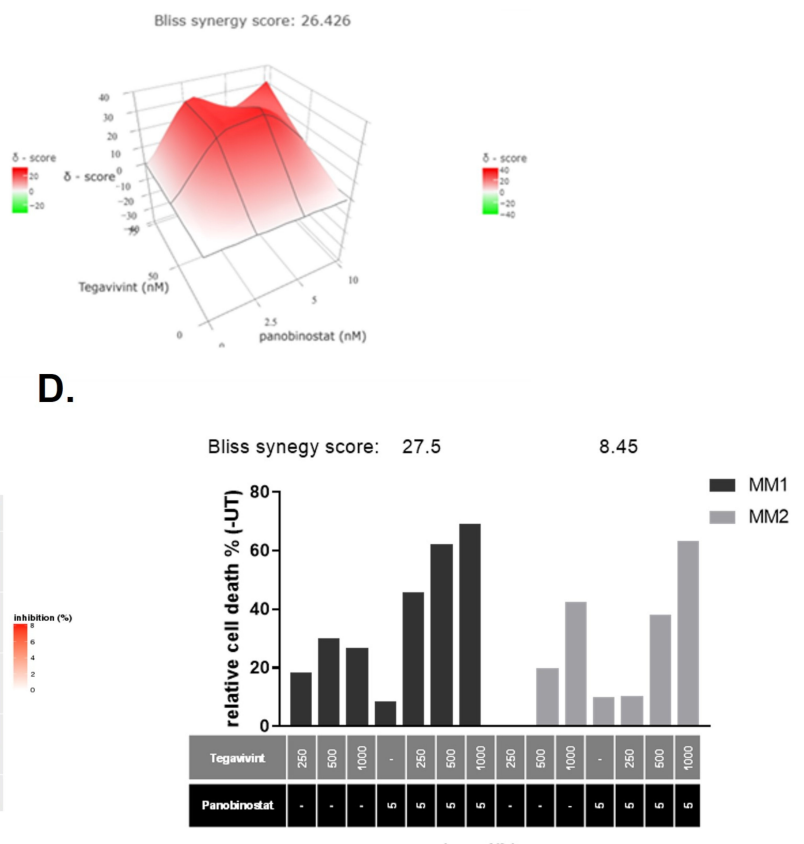

(nmol/L)

Figure 1. The combination of Tegavivint and panobinostat exerts synergistic anti-MM effects in vitro and ex vivo. (A) Seven HMCL were treated with clinically relevant doses of panobinostat (up to $20 \mathrm{nmol} / \mathrm{L})$ for $48 \mathrm{~h}$ and PI+ was measured by FC $(n=3-6)$. Cell viability was calculated as a relative percentage compared with vehicle alone (100\%). U266 were further treated with increasing doses of panobinostat up to $200 \mathrm{nmol} / \mathrm{L}$ for $48 \mathrm{~h}$ and propidium iodide $+(\mathrm{PI}+)$ was measured by flow cytometry (FC) $(n=6)$. (B) NCIH929, OCIMy1, and U266 were treated with Tegavivint, panobinsotat, or their combination for $48 \mathrm{~h}$. PI+ was measured by FC $(n=3)$. The expected drug combination responses were calculated based on Bliss reference model using SynergyFinder. Deviations between observed and expected responses with positive and negative values denote synergy and antagonism respectively. (C) Non-malignant HS5 cells were treated with similar doses of Tegavivint or panobinostat alone for $48 \mathrm{~h}$ and PI+ was measured by FC. Cell viability was calculated as a relative percentage compared with vehicle alone $(100 \%)(n=3)$ (left panel). HS5 cells were treated with Tegavivint, panobinsotat, or their combination for $48 \mathrm{~h}$ and PI+ was measured by flow cytometry (FC) $(n=3)$. Dose response matrix was produced (right panel). (D) Proportion of apoptotic (Apo $2.7+) \mathrm{CD} 38^{+} \mathrm{CD} 45^{-}$primary MM cells in an autologous bone marrow coculture assay after $72 \mathrm{~h}$ of Tegavivint, panobinostat, or the combination. Numbers represent calculated Bliss synergy scores for each patient sample. 
3.2. The Combination of Tegavivint and Panobinostat Induces Apoptosis and Downregulates Wnt Pathway Down-Stream Targets

We quantified apoptosis induction in OCIMy1 and U266 by annexin V, annexin $\mathrm{V} / 7 \mathrm{AAD}$ to the combination of Tegavivint (50-75 $\mathrm{nmol} / \mathrm{L})$ and panobinostat $(5-10 \mathrm{nmol} / \mathrm{L})$. Apoptosis initiation and commitment of cells to late apoptosis (annexin V+/7AAD+) were apparent for OCIMy1 at $24 \mathrm{~h}$ whereas for U266 they were significantly augmented at $48 \mathrm{~h}$ (Figure 2A, Supplementary Figure S3A), while there was no significant increase of annexin $\mathrm{V}-/ 7 \mathrm{AAD}+$ cells (indicative of non-apoptotic cell death). These findings were consistent with apoptotic activation being one of the main mediators of cell death induced by the combination. Accordingly, the levels of active caspase 9 (a measure of the intrinsic apoptotic pathway activity) were elevated at the same timepoints $(46 \%$ for OCIMy 1 treated with $75 \mathrm{nmol} / \mathrm{L}$ of Tegavivint and $10 \mathrm{nmol} / \mathrm{L}$ panobinostat, and 47\% for U266 treated with the same combination) (Figure 2B, Supplementary Figure S3B).

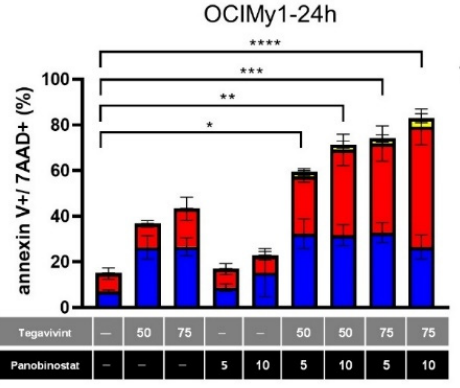

B.

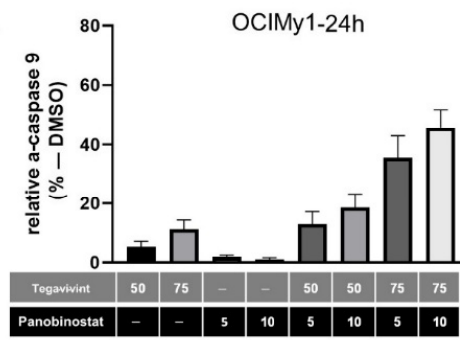

C.

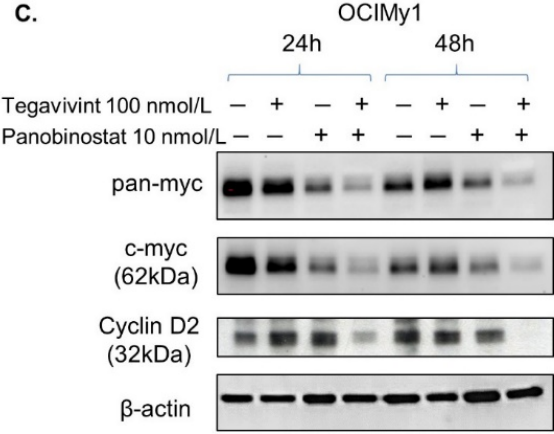

U266-48h

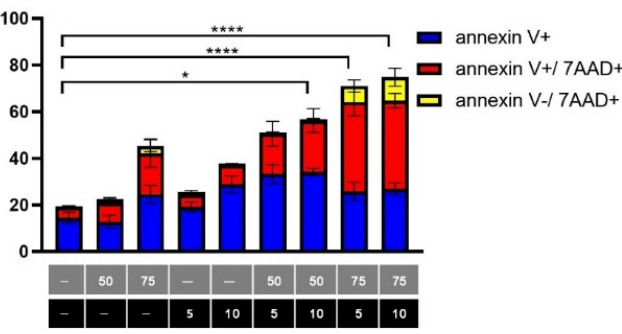

U266-48h

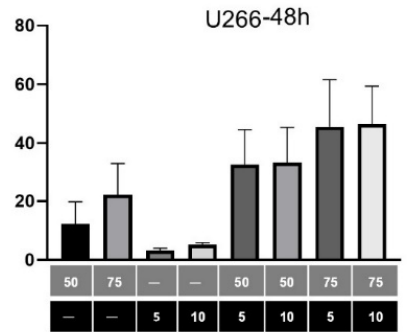

Figure 2. The combination of Tegavivint and panobinostat induces apoptotic cell death and downregulation of $\beta$-catenin targets. (A) Percentage of annexin $V$, annexin V/7AAD, and 7AAD-positive cells after Tegavivint, panobinostat or the combination for OCIMy1 and U266 at 24 and $48 \mathrm{~h}$ respectively ( $n=3, \pm S E)$. 2way ANOVA Dunett's multiple comparisons test of row means to control row mean (DMSO treated) ${ }^{*} p=0.0175,{ }^{* *} p=0.0013,{ }^{* * *} p=0.0007,{ }^{* * * *} p<0.0001$ for OCIMy1 and ${ }^{*} p=0.0164,{ }^{* * * *} p<0.0001$ for U266). (B) Percentage of active caspase-9 after treatment with Tegavivint, panobinostat, or the combination for OCIMy1 and U266 at 24 and $48 \mathrm{~h}$ respectively relative to the vehicle (DMSO) treated cells $(n=3, \pm \mathrm{SE})$. (C) Immunoblotting of whole-cell lysates for $\beta$-catenin down-stream targets Myc, Cyclin D1 and Cyclin D2 after 24 or $48 \mathrm{~h}$ of treatment with Tegavivint, panobinostat, or the combination for OCiMy1 and U266. Loading control: $\beta$-actin $(n=3)$. Detailed information about the Western blotting can be found at Figure S4. 
Cyclin D and Myc dysregulation plays a critical role in the pathogenesis of MM [17] and both are down-stream targets of the canonical Wnt pathway [11]. Combination treatment of OCIMy1 (expressing high Myc and Cyclin D2 at baseline) and U266 (expressing high L-Myc and Cyclin D1 at baseline) induced significant Myc and Cyclin D downregulation as early as $24 \mathrm{~h}$ after treatment (Figure 2C, Supplementary Figure S4).

Collectively, our results confirm the synergistic pro-apoptotic effect of the combination and its significant negative impact on the expression of MM promoting oncogenes.

\subsection{Tegavivint in Combination with Panobinostat Targets the Mitochondria Fitness of MM Cells}

There is an increasing body of evidence implicating Myc in the metabolic reprogramming of cancer cells [18,19] whereas HDACi have been shown to alter glucose metabolism in MM cells [20]. In this context, having shown down-regulation of Myc protein expression with the combination of Tegavivint and panobinostat, we evaluated their effect on the mitochondrial fitness and metabolic activity of OCIMy1 and U266 cells.

The MFI (median fluorescent intensity) of TMRE (measure of transmembrane potential/mitochondrial fitness) fell significantly upon the addition of $100 \mathrm{nmol} / \mathrm{L}$ Tegavivint with 5 or $10 \mathrm{nmol} / \mathrm{L}$ of panobinostat for $20 \mathrm{~h}(p=0.001$ and $p=0.001$, and $p=0.05$ and $p=0.03$, for OCIMy1 and U266, respectively) (Figure 3A). Consistent with this observation, analysis of TMRE by confocal microscopy in combination with MitoTracker Green FM staining similarly demonstrated the deleterious impact of the combination on the mitochondria fitness of both cell lines (Figure 3B, Supplementary Figure S5A). Although Wnt signalling and consequently $\beta$-catenin have been shown to strongly activate mitochondrial biogenesis [21,22] the combination of Tegavivint and panobinostat did not numerically alter the mitochondria, as measured by MitoTracker Green FM staining and FC, during the time frame of the described experiments (Supplementary Figure S5B).

To further study the effect of Tegavivint and panobinostat on cellular metabolism we conducted extracellular flux analysis. We assessed the effect of single drugs (Tegavivint $100 \mathrm{nmol} / \mathrm{L}$ and panobinostat $10 \mathrm{nmol} / \mathrm{L}$ ) and the combination on glycolysis by determining ECAR (extracellular acidification rate) in the context of a glycolysis stress test (Figure 4A). Consistent with the known effects of HDACi on aerobic glycolysis [20] panobinostat was able to significantly suppress glycolysis in both cell lines (measures derived from the measurement of ECAR) as a single agent, with the effect further augmented by the addition of Tegavivint in terms of glycolytic capacity (Supplementary Figure S6). Knowledge on the effects of Myc and/or panobinostat treatment on oxidative phosphorylation (oxphos) is limited. To this end, we assessed the effect of single drugs and the combination on oxphos by measuring the OCR (oxygen consumption rate) in the context of a mitochondrial stress test (Figure 4B). This revealed that both basal respiration and ATP production were significantly reduced by the combination treatment $-p=0.0001$ and $p=0.0001$, respectively, for OCIMy1, and $p<0.0001$ and $p=0.0004$, respectively, for U266. Moreover, for U266, which demonstrated higher respiratory capacity, maximal respiration was also significantly reduced by the combination $(p=0.0002)$ (Figure $4 \mathrm{C})$. Collectively, these data demonstrate that in MM cells the combination of Tegavivint and panobinostat markedly inhibits oxidative phosphorylation and to a lesser extent aerobic glycolysis thus impairing metabolic pathways essential for energy and biomolecule generation. 
A.

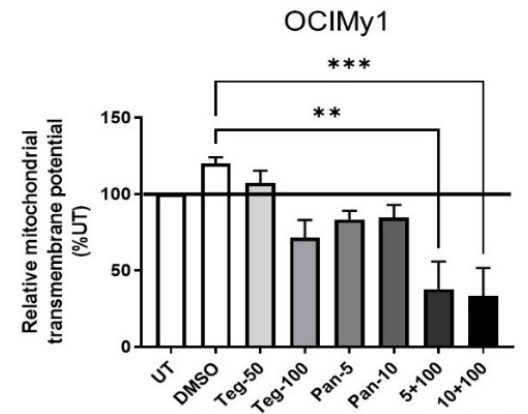

B. OCIMy
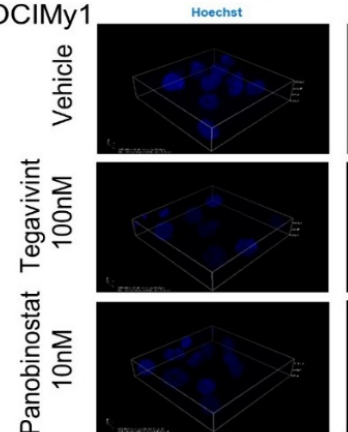

है

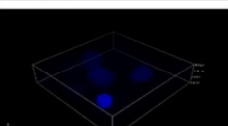

导
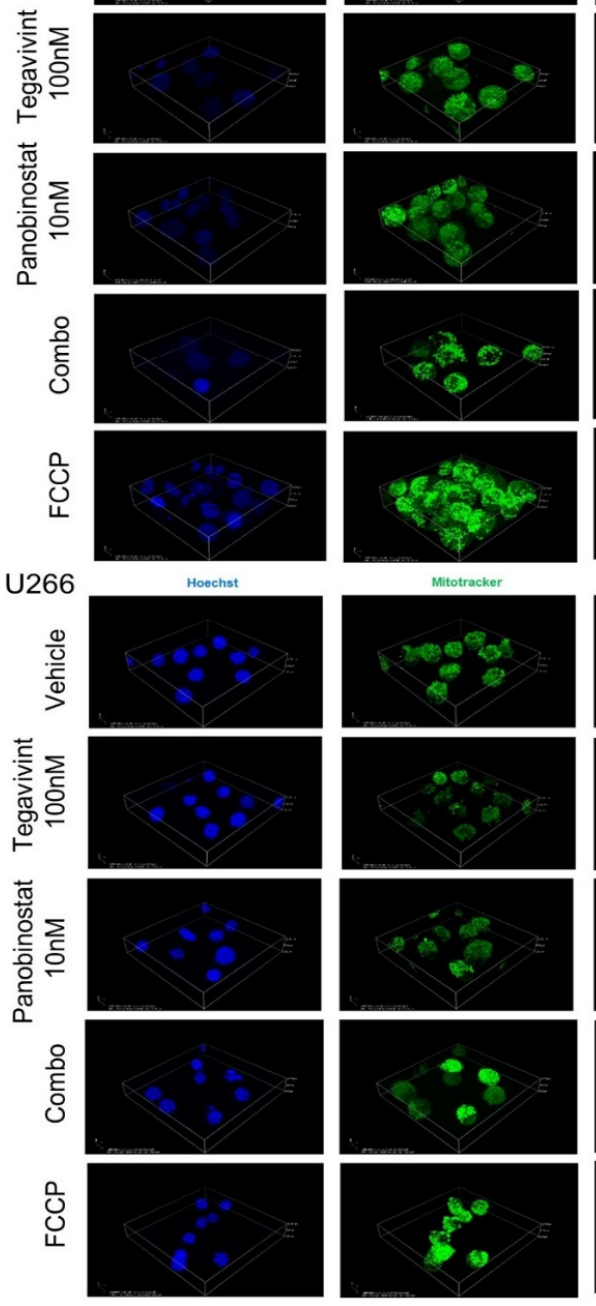
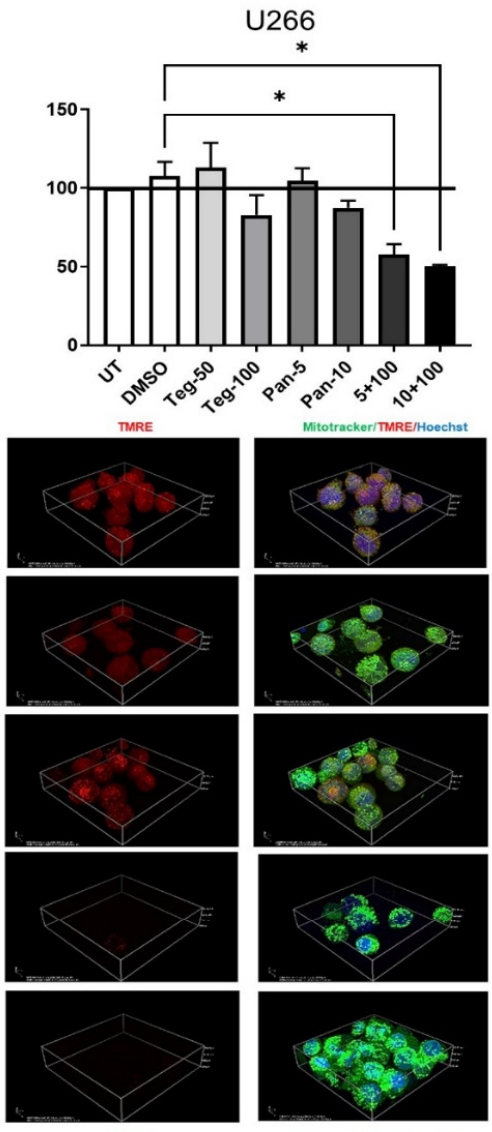

MRE
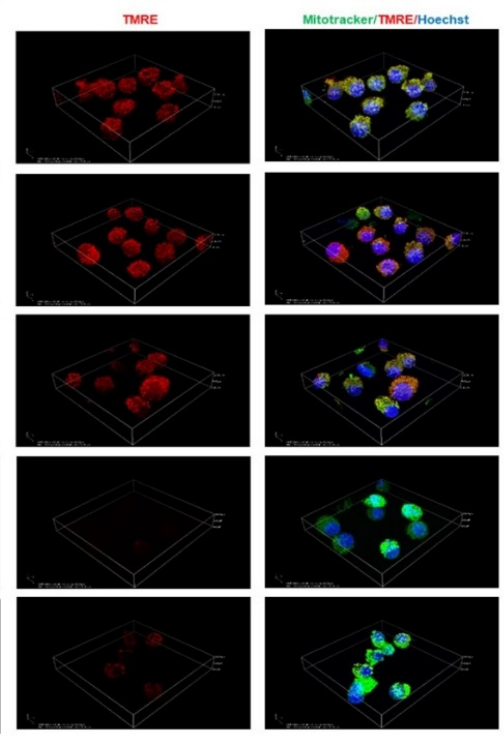

Figure 3. Tegavivint in combination with panobinostat targets mitochondrial fitness of MM cells. (A) OCIMy1 and U266 cells were treated with Tegavivint (Teg-), panobinostat (Pan-), Tegavivint and panobinostat, or vehicle (DMSO) for $20 \mathrm{~h}$. Mitochondrial transmembrane potential was measured by TMRE staining and FC. MFI was measured and expressed as a relative \% to the TMRE MFI of UT (untreated) cells (UT $=100 \%)(n=3, \pm \mathrm{SE}$, nonparametric Kruskal-Wallis multiple comparisons test, ${ }^{* *} p=0.0013,{ }^{* * *} p=0.001$ for OCIMy1, and ${ }^{*} p=0.04$ and 0.03 for U266 respectively). (B) OCIMy1 and U266 were treated with Tegavivint, panobinostat or the combination for $20 \mathrm{~h}$ and stained with TMRE (red for mitochondrial potential), Mito Tracker Green (green for mitochondria) and Hoechst 33,342 (blue for nuclei) and evaluated with confocal microscopy. One $\mu \mathrm{mol} / \mathrm{L}$ of the uncoupling agent FCCP was added $20 \mathrm{~min}$ before imaging and used as a positive control (magnification $40 \times \mathrm{WI}$ ). 

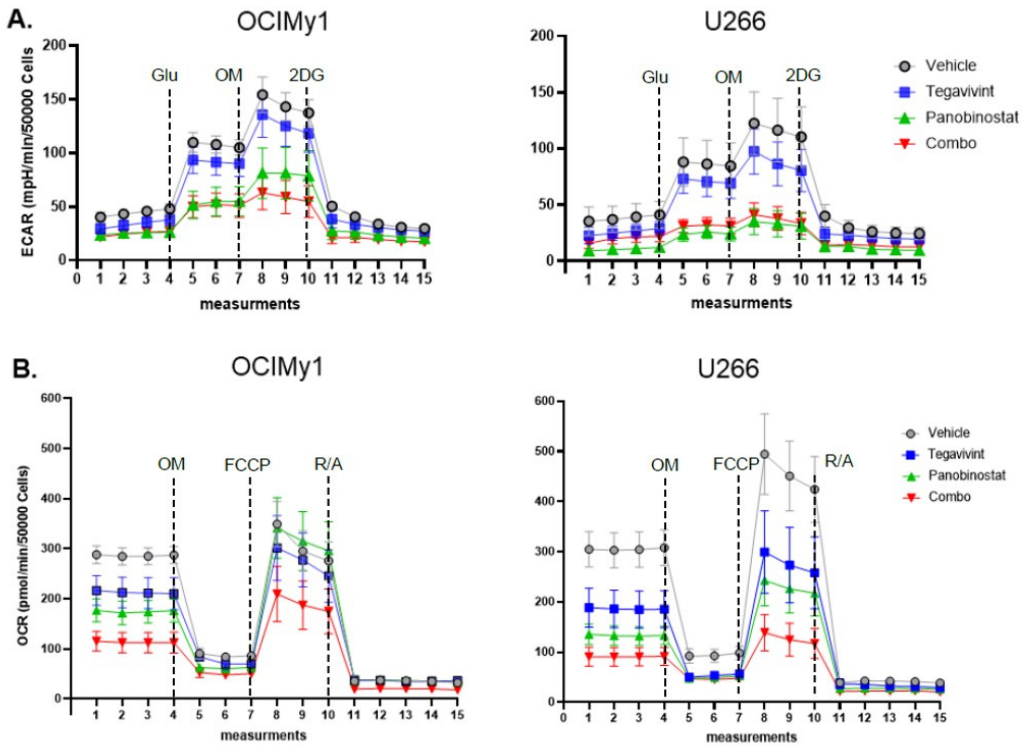

c.
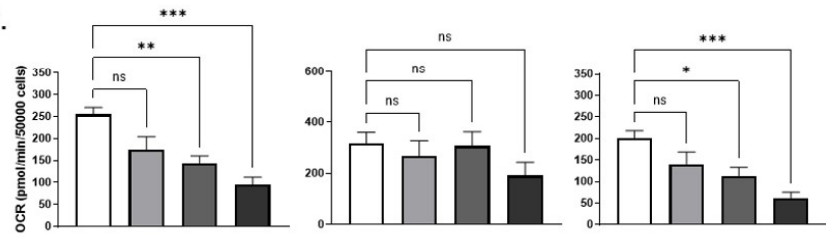

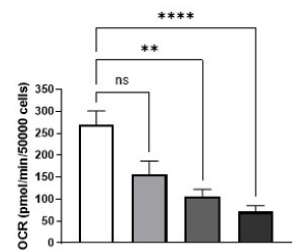

Basal respiration

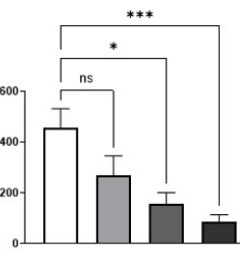

Maximal respiration

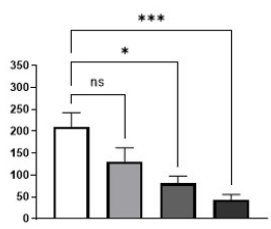

ATP production
ㅁ Tegavivint
- Panobinostat OCIMy1

U266

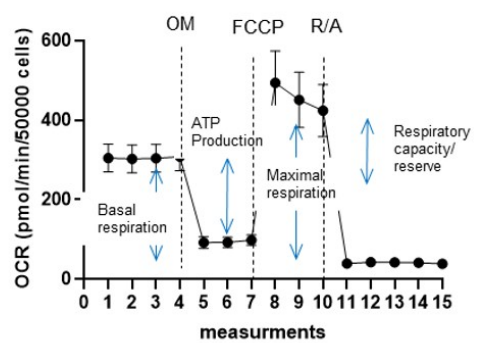

Figure 4. Tegavivint in combination with panobinostat reduces oxidative phosphorylation of MM cells. (A) OCIMy1 and U266 cells were treated with vehicle, $100 \mathrm{nmol} / \mathrm{L}$ of Tegavivint, $10 \mathrm{nmol} / \mathrm{L}$ of panobinostat, or the combination for $18 \mathrm{~h}$. Analysis of ECAR (extracellular acidification rate) was performed using a Seahorse XF analyser to assess glycolysis in the context of a glycolysis stress test. Three technical replicates were performed for each experimental variable $(n=3, \pm \mathrm{SE})$ (Glu: glucose, OM: oligomycin, 2DG: 2-deoxy-D-glucose). (B) OCIMy1 and U266 cells were treated similarly to Figure $3 \mathrm{~A}$ for $18 \mathrm{~h}$. Analysis of OCR (oxygen consumption rate) was performed using a Seahorse XF analyser to assess mitochondrial respiration/oxphos (oxidative phosphorylation) in the context of a mitochondrial stress test. Three technical replicates were performed for each experimental variable ( $n=3, \pm \mathrm{SE}$ ) (OM: oligomycin, FCCP: carbonyl cyanide 4-(trifluoromethoxy)phenylhydrazone R/A: rotenone/antimycin). (C) Results from the OCR analysis were further analysed and the basal respiration, maximal respiration, and ATP production for OCIMy1 and U266 were calculated $(n=3$, $\pm \mathrm{SE}$ ) (nonparametric Kruskal-Wallis multiple comparisons test ${ }^{*} p \leq 0.05,{ }^{* *} p \leq 0.01,{ }^{* * *} p \leq 0.001$, $* * * * p \leq 0.001)$. 
3.4. Tegavivint Overcomes Acquired Panobinostat Resistance in Secondary Bortezomib Resistant MM Cells

A recent meta-analysis evaluating the efficacy and safety of $\mathrm{HDACi}$, including panobinostat, in RRMM, demonstrated that patients who were bortezomib-refractory were less likely to benefit from HDACi [23]. To investigate the effect of Tegavivint and panobinostat in the context of proteasome-inhibitor (PI) resistance we generated KMS12PE cells resistant to both carfilzomib (KR) and bortezomib (VR). Although the KR and parental cells both remained sensitive to bortezomib $\left(\mathrm{LD}_{50}=8.3 \mathrm{nmol} / \mathrm{L}\right.$ and $12.9 \mathrm{nmol} / \mathrm{L}$, respectively), the VR cell line showed a 20-fold increase in bortezomib $\mathrm{LD}_{50}\left(\mathrm{LD}_{50}=200 \mathrm{nmol} / \mathrm{L}\right)$ confirming the acquisition of secondary bortezomib resistance (Figure 5A). PI resistance was accompanied by relative panobinostat resistance in both resistant cell lines $\left(\mathrm{LD}_{50}=96.1 \mathrm{nmol} / \mathrm{L}\right.$ for VR and $82.9 \mathrm{nmol} / \mathrm{L}$ for KR vs. $\mathrm{LD}_{50}=27.8 \mathrm{nmol} / \mathrm{L}$ for the parental cells) (Figure 4B-left panel). Although there was no difference of sensitivity to Tegavivint amongst parental and VR cells $\left(\mathrm{LD}_{50}=84.8 \mathrm{nmol} / \mathrm{L}\right.$ vs. $\mathrm{LD}_{50}=86.4$ for the VR and parental cells, respectively), $\mathrm{KR}$ cells showed a significant increase of sensitivity $\left(\mathrm{LD}_{50}=46.2 \mathrm{nmol} / \mathrm{L}\right)$ (Figure $5 \mathrm{~B}$-right panel). Importantly, Tegavivint at concentrations ranging from $25 \mathrm{nmol} / \mathrm{L}$ to $75 \mathrm{nmol} / \mathrm{L}$ was able to re-sensitise VR cells to low panobinostat concentrations ( 5 and $10 \mathrm{nmol} / \mathrm{L}$ ), and further augment the synergistic effect of the two drugs as shown by an increased Bliss synergy score (17.0) compared to the corresponding score for the parental cells (12.0) (Figure 5C).

A.

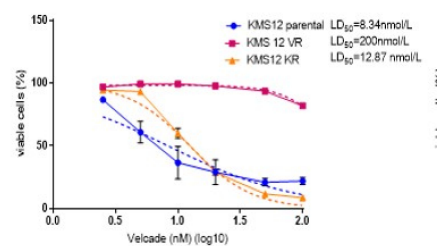

C.

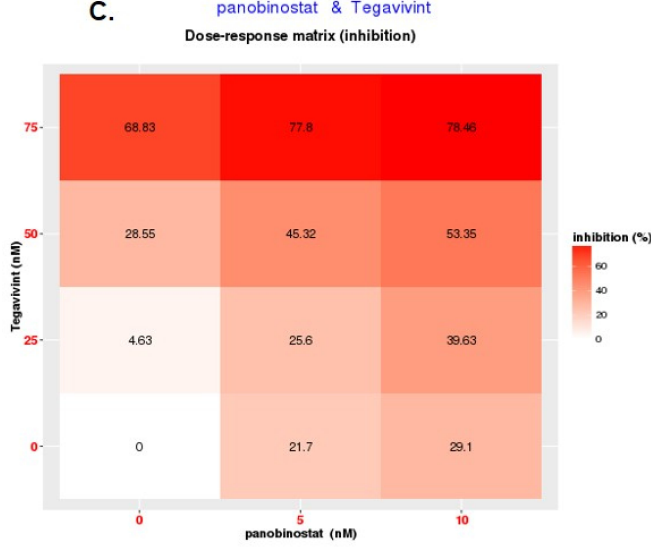

Tegavivint (nM) \& panobinostat $(n M)$
Blliss synergy score: 11.539

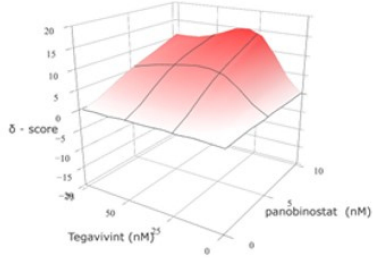

KMS12PE parental

B.
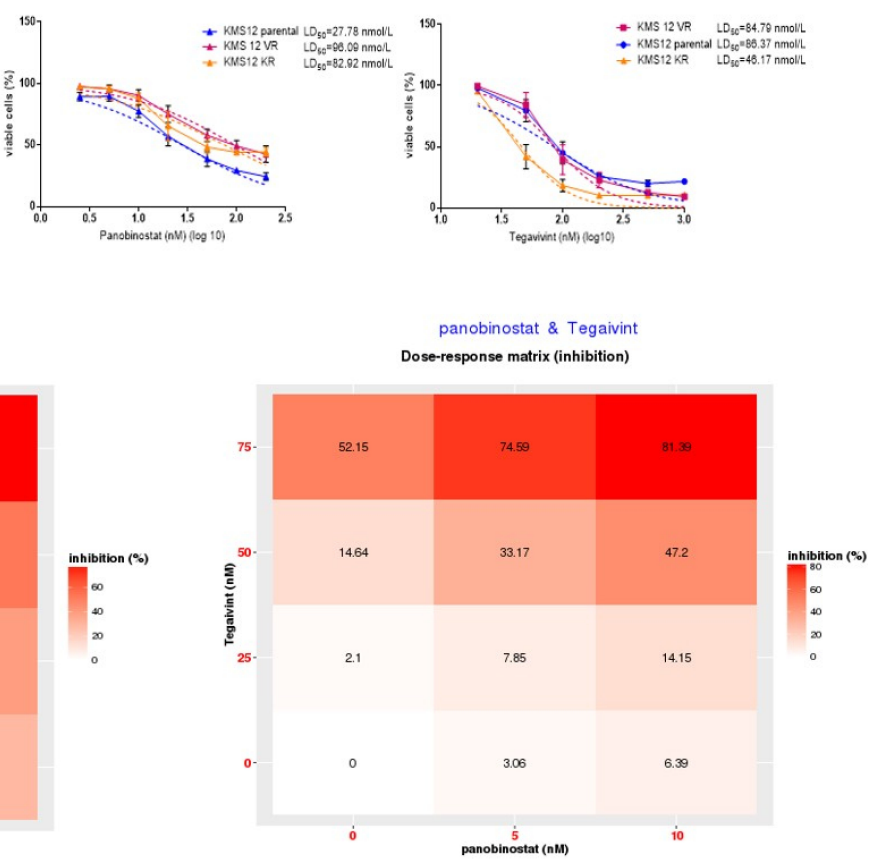
Tegaivint $(\mathrm{nM})$ \& panobinostat $(\mathrm{nM})$
Bliss synergy score: 16.976

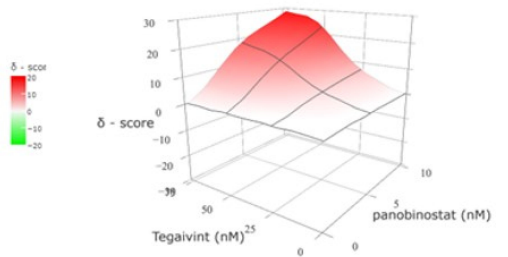

KMS12PE VR

Figure 5. Tegavivint overcomes acquired panobinostat resistance in bortezomib resistant MM cells. 
The HMCL KMS12PE was cultured for a 12 months period in slowly increasing concentrations of bortezomib (VR), carfilzomib (KR), or vehicle (parental). Resistant cells were stored for 6 months, thawed and cultured for an additional 12 week period in the absence of drugs to confirm the acquisition of permanent drug resistance. (A). Cells were treated with increasing doses of bortezomib or carfilzomib for $48 \mathrm{~h}$ and cell viability was calculated after PI staining and FC (\% of vehicle treated cells). (B). Parental, VR and KR cells were treated with increasing doses of panobinostat for $48 \mathrm{~h}$ and cell viability was calculated after PI staining and FC (\% of UT) (left panel). Parental, VR and KR cells were treated with increasing doses of Tegavivint for $48 \mathrm{~h}$ and cell viability was calculated after PI staining and FC (\% of UT) (right panel). For calculations of LD 50 we applied the non-linear regression model. Dashed lines correspond to the non-linear fit curves. (C). Parental and VR cells were treated with Tegavivint, panobinostat or the combination for $48 \mathrm{~h}$ and the PI+ was measured by FC $(n=3)$. Dose response matrices were produced for both cell lines and the expected drug combination responses were calculated based on the Bliss reference model using SynergyFinder. Deviations between observed and expected responses with positive and negative values denote synergy and antagonism respectively.

These results suggest that the combination of Tegavivint and panobinostat may be able to overcome the therapy resistant phenotype in RRMM patients with primary or secondary bortezomib refractoriness.

\subsection{Tegavivint in Combination with Panobinostat Exerts Potent Anti-MM Effects In Vivo}

We assessed the in vivo activity of low dose Tegavivint and panobinostat both as single agents and in combination using a biweekly dosing schedule, for both panobinostat and Tegavivint, in a systemic myelomatosis xenograft utilising U266 cells, for a total of two cycles. Panobinostat was administered at $10 \mathrm{mg} / \mathrm{kg}$ biweekly, while the approved dose for the treatment of patients with RRMM is $20 \mathrm{mg} / \mathrm{kg}$, three times weekly [5]. Tegavivint was administered at $30 \mathrm{mg} / \mathrm{kg}$, while $50 \mathrm{mg} / \mathrm{kg}$ have been shown to exert anti-tumour effect in a solid tumour mouse model [10]. The animals were treated for the first three weeks of each of two 28-day cycles, so, three weeks on one week off. With the combination, significant inhibition of tumour growth, as measured by luciferase bioluminescence, was evident by day 14 of cycle 1 ( $p=0.02$ ). After the last administration of drugs (day 49) (Figure 6A left panel) the reduction in tumour burden with the combination when compared to vehicle control, Tegavivint, or panobinostat was highly statistically significant $(p=0.0003)$ (Figure 6A right panel). Moreover, and despite the limited number of therapeutic cycles the inhibition of tumour growth induced by the combination translated into a significant prolongation in survival ( $p=0.01$ ) (Supplementary Figure S7). 

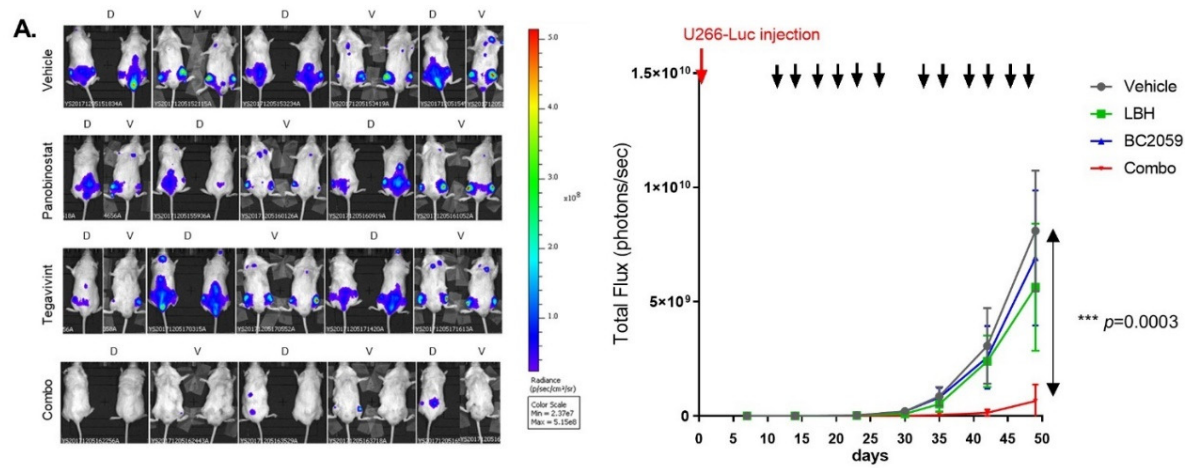

B.

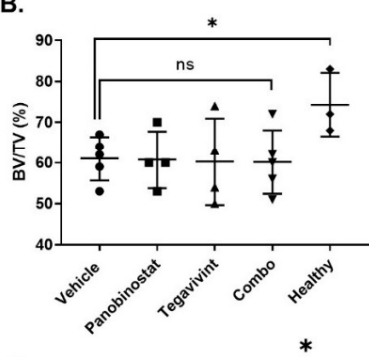

C.
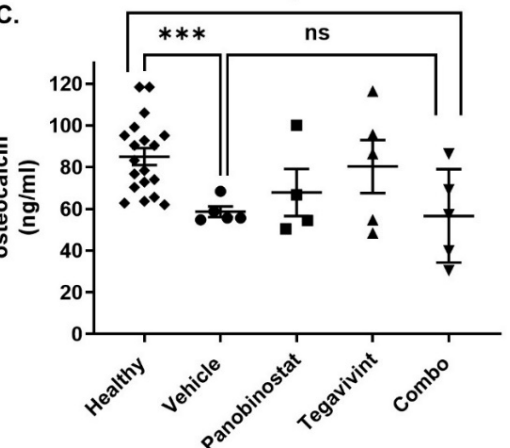
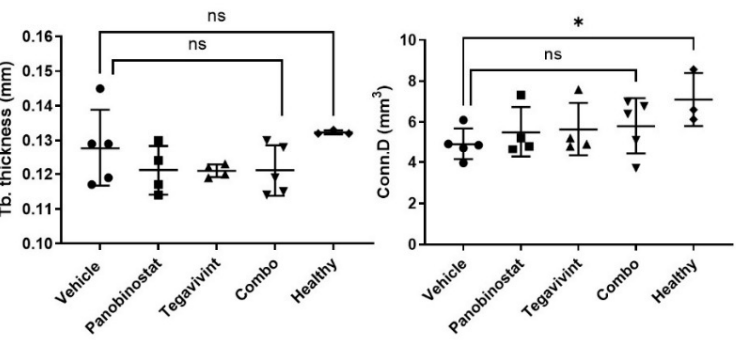

D.

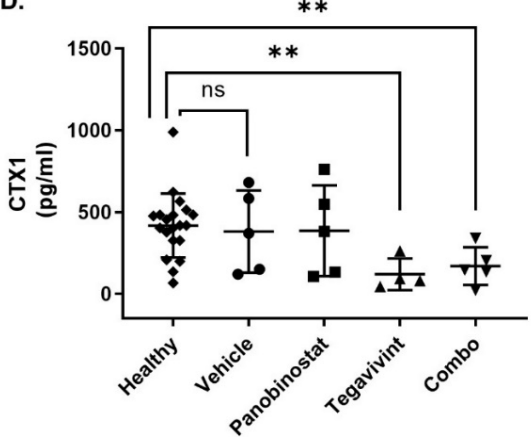

Figure 6. Tegavivint in combination with panobinostat exerts potent anti-MM effect in vivo, without a negative impact on bone metabolism. (A) NSG human MM-bearing mice received vehicle (17\% Solutol HS 15 (Sigma-Aldrich) and normal saline) $(n=5)$, whereas treated mice received $10 \mathrm{mg} / \mathrm{kg}$ panobinostat $(n=5)$ or $30 \mathrm{mg} / \mathrm{kg}$ Tegavivint $(n=5)$, or their combination $(n=5)$ twice a week intraperitoneally for 3 consecutive weeks for 2 cycles of 28 days each. Tumour growth was monitored weekly with in vivo bioluminescence imaging. Dorsal (D) and ventral (V) views of NSG human MM-bearing mice at day 49 (end of cycle 2 treatment). The median flux (photons/second) of dorsal and ventral views for each cohort is documented ( $n=5$ for each cohort, $\pm \mathrm{SD}$ ). Multiple $t$-tests were used for statistical analysis without assuming SD, and statistical significance was determined using the Holm-Sidak method, with $\alpha=5.000 \%$. $\downarrow$ : therapeutic intervention. (B) Adult NSG human MMbearing mice were treated as mentioned above for two cycles. Upon reaching experimental end point mice were humanely euthanised and micro-CT of L5 vertebrae was performed. BoneJ was used for the analysis and the calculation of bone morphometric indices and compared with the measurements derived from three healthy age matched NSG mice. Treatment with single drugs or the combination did not negatively affect the bone volume fraction (BV/TV), trabecular thickness (Tb. Thickness) or the connectivity density (Conn. D) ${ }^{*} p \leq 0.05$, nonparametric Mann-Whitney $t$ test). (C) During the last week of cycle 2 serum was analysed for osteocalcin levels by ELISA. Derived values were compared with serum osteocalcin concentrations before the injection of U266-MM cells (healthy) (*** $p=0.0005$ and $* p=0.02$, nonparametric Mann-Whitney $t$ test). (D) Similarly, serum was analysed for carboxy-terminal collagen crosslinks (CTX1) levels by ELISA. Derived values were compared with serum CTX1 concentrations before the injection of U266-MM cells (healthy) $\left({ }^{* *} p=0.005\right.$ and ** $p=0.008$ for healthy versus Tegavivint or combination, respectively, nonparametric Mann-Whitney $t$-tests). 


\subsection{Tegavivint in Combination with Panobinostat Is Well Tolerated In Vivo}

The pivotal role of the Wnt canonical pathway in haemopoietic stem cell (HSC) maintenance [24] has raised theoretical concerns in relation to the therapeutic applicability of Wnt pathway inhibitors. In our in vivo experiment biweekly dosing for two cycles while exhibiting significant anti-MM activity was not associated with weight loss (Supplementary Figure S8A, left panel) consistent with the maintenance of intact gastro-intestinal function. Furthermore, immune-histochemical (IHC) studies of the colon harvested at the time of euthanasia demonstrated normal histologic features in the combination treated mice (Supplementary Figure S8A, right panel). Moreover, combination was not associated with peripheral blood cytopenias at any of the evaluation time-points (Supplementary Figure S8B).

\subsection{Tegavivint in Combination with Panobinostat Does Not Negatively Impact Bone Metabolism In Vivo}

The Wnt pathway is recognised as being a key regulator of bone homeostasis [25]. This is of particular relevance in MM as almost $80 \%$ of newly diagnosed MM patients have evidence of significant bone loss due to dysregulation of complex and not yet fully understood interactions between osteoblasts, osteoclasts, osteocytes, bone matrix, and immune cells [26]. Considering the engraftment of U266 in the bone marrow of the spine (especially lumbar) and long bones (Figure 6A) and in order to study the potential impact of Tegavivint on bone homeostasis, we performed micro-CT of the 5th lumbar vertebrae from the xenografts harvested at the time of euthanasia. Micro-CT results were compared to those from three healthy control NSG mice of similar age. While the presence of MM disease was associated with a significant decrease in bone volume fraction BV/TV (BV: bone volume, TV: total volume of interest) $(p=0.04)$ no additive bone loss was observed in the animals treated with Tegavivint or with Tegavivint in combination with panobinostat (Figure 6B).

Serum levels of osteocalcin (a measure of osteoblastic activity) and CTX1 (carboxyterminal collagen crosslinks) (a measure of osteoclastic activity) were measured at baseline (before the injection of MM cells) (healthy) and at the last week of treatment of cycle 2. A reduction in osteocalcin (healthy vs. vehicle, $p<0.001$ ), representative of MM-induced inhibition of osteoblastic activity was consistent with the micro-CT findings but importantly there was no evidence of additional inhibition of osteoblastic activity related to Tegavivint (Figure 6C). Interestingly, CTX1 levels at the same time point showed a significant decrease in osteoclastic activity in the Tegavivint alone and Tegavivint and panobinostat combination treated animals when compared to the healthy control levels, the mechanism of which is unclear (Figure 6D).

\section{Discussion}

Panobinostat is the only HDACi approved in the USA and Europe for the treatment of RRMM [5]. Approval was based on the results of the PANORAMA 2 TRIAL which paradoxically demonstrated a relatively greater survival benefit for the most heavily pretreated patients receiving panobinostat in combination with intra-venous bortezomib and dexamethasone, likely reflecting the unique and pleiotropic effects of HDACi on MM cells [4]. The combination was, however, associated with a challenging toxicity profile that is largely mitigated with the use of sub-cutaneous bortezomib and alternative panobinostat scheduling as demonstrated in the recently published PANORAM 3 trial [5]. This fact, notwithstanding the role and optimal combinatorial approach for panobinostat use in MM, remains unclear.

Several lines of evidence have confirmed the presence of an active intrinsic Wnt canonical signalling in MM [8] as a result of both genetic and epigenetic abnormalities [23], with greater dysregulation evident, coincident with disease progression [27]. Significantly, upregulation of the Wnt pathway is also thought to be an important mechanism driving lenalidomide resistance [28] further supporting the exploration and validation of Wnt 
pathway inhibitors for the treatment of RRMM. Interactions of $\beta$-catenin-HDAC have long been recognised $[29,30]$, and a dual targeting approach has been successfully validated in AML [11]. In this work we showed that low doses of the $\beta$-catenin inhibitor Tegavivint can augment the anti-MM effect of low dose panobinostat, by initiating intrinsic apoptosis, even in the highly resistant U266. This cell line despite carrying $\mathrm{t}(11 ; 14)$, which recently has been shown to respond to $\mathrm{Bcl} 2$ inhibitor venetoclax [31], is resistant to the latter [32], rendering it a difficult to kill cell line. Dual inhibition was able to significantly down-regulate known targets of the Wnt canonical pathway, namely Cyclin D1, Cyclin D2, and Myc. Myc is a well-recognised pro-survival factor and master regulator of glycolysis [18], whereas HDAC inhibitors have been shown to affect aerobic glycolysis [20]. Accordingly, panobinostat, was able to reduce the aerobic glycolytic activity of MM cells, as measured by ECAR, without though inducing significant cell death, whereas only the combination was able to negatively impact oxidative phosphorylation, measured by OCR. The significant decrease of OCR and loss of mitochondrial transmembrane potential was shown to be due to mitochondrial damage and not due to decreased mitochondria biogenesis. Thus, combination of both drugs was capable of compromising both energy production machineries leading to MM cell apoptotic cell death.

Bortezomib refractory patients were only included in the PANORAMA 2 study while excluded in the PANORAMA 1 and 3 [5]. A meta-analysis of panobinostat treated patients showed an overall response rate of $32 \%$ for those refractory to bortezomib and $43 \%$ for those refractory to lenalidomide suggesting that panobinostat had superior activity in the latter subgroup [22]. In our study we were able to confirm the acquisition of panobinostat resistance upon acquiring bortezomib or carfilzomib resistance in vitro and that the addition of Tegavivint was able to reverse panobinostat resistance in the former, underpinning the adoption of alternative combination for the treatment of patients with PI resistance. The mechanisms of panobinostat resistance in these cell lines are currently being investigated.

One of the aims of the recently published PANORAMA 3 was to assess alternative scheduling and dosing of panobinostat to mitigate its toxicity. Although the regimen with the highest dose and frequency was the most effective, lower doses (10 $\mathrm{mg}$ ) proved to be better tolerated. Similarly, for the in vivo validation of the proposed combination we used low dose panobinostat $(10 \mathrm{mg}$ ) administered twice weekly in combination with low dose Tegavivint with no significant toxicity seen after the completion of two cycles of treatment. Our scheduling, as shown by the tumour growth inhibition benefit, was able to exert significant anti-tumour effect while minimising potential toxicity.

The central role of the Wnt canonical pathway in orchestrating homeostasis of various tissues, including adult bone, has hindered the development of Wnt pathway inhibitors. Interestingly, the results of several studies evaluating monoclonal antibodies and small molecule inhibitors in a variety of Wnt driven tumours have been promising, with the majority of these molecules, including Tegavivint, being well tolerated in the models utilised [9-12]. The lack of toxicity is believed to be due to the 'just-right' model of Wnt pathway activation, which suggests that each tissue, including tumour cells, have an optimal and different threshold of Wnt activation [23,33]. Accordingly, in our in vivo model the combination treatment did not negatively affect haemopoiesis, GI tract epithelium, or bone homeostasis validated at the end of the experiment (different time point for each mouse). Additionally, at these low dosages Tegavivint alone or in combination with panobinostat at the end of the 2nd cycle did not further inhibit osteoblastic activity but intriguingly down-regulated osteoclastic activity. The reduced osteoclastic activity cannot be explained by differences in tumour burden as tumour growth in the Tegavivint alone cohort did not differ significantly from the vehicle cohort. One possible explanation could be decreased secretion of DKK1 by MM cells. DKK1, a Wnt pathway inhibitor is a downstream target of $\beta$-catenin [34], known to promote osteoclastic activity, while inhibiting osteoblastic differentiation [35], thus chemical inhibition of the pathway could lead to down-regulation of its transcription, but this hypothesis would require further evaluation. 
It is well known that the Wnt signaling pathway has been implicated in multiple aspects of MM disease, namely disease progression and acquisition of drug resistance as well as imbalance between bone-forming osteoblasts and bone-resorbing osteoclast, leading to the characteristic osteolytic bone lesions of MM patients [8]. Thus, targeting the Wnt pathway could prove to be an attractive avenue, especially for patients who have acquired resistance to mainstream therapies, without further deteriorating their bone disease, as concluded from our in vivo experiment. Nevertheless, taking into consideration the central role of the Wnt pathway in regulating tissue-specific stem cell populations [36], close monitoring of blood counts and GI tract condition is suggested for patients with RRMM receiving the aforementioned combination.

\section{Conclusions}

Novel drugs and drug combinations have undoubtfully changed the paradigm for the treatment of MM, leading to significant prolongation of both PFS and OS. Nonetheless, the majority of patients will sequence through multiple relapses exhausting all possible treatments, through the acquisition of drug resistance to the mainstream therapies. We have shown in this study that panobinostat, in combination with Tegavivint has a significant anti-MM effect with a favourable toxic profile and may prove to be a beneficial alternative for the treatment of highly pre-treated RRMM that warrants further evaluation.

Supplementary Materials: The following are available online at https://www.mdpi.com/article/10 .3390/ cancers14030840/s1, Figure S1: (A) NCIH929 and OCIMy1 HMCL were treated with increasing doses of panobinostat for $48 \mathrm{~h}$ and cell viability was calculated after PI staining and FC, (B) NCIH929, OCIMy1 and U266 HMCL were treated with increasing doses of Tegavivint for $48 \mathrm{~h}$ and cell viability was calculated after PI staining and FC, Figure S2: (A) Calculation of Bliss synergy score for primary MM cells in an autologous bone marrow coculture assay after $72 \mathrm{~h}$ of Tegavivint, panobinostat, or their combination (B) Calculation of Bliss synergy score for primary stromal cells in the aforementioned experiment, Figure S3: (A) Percentage of annexin V, annexin V/7AAD, and 7AAD positive cells after vehicle alone (DMSO), Tegavivint (50 and $75 \mathrm{nmol} / \mathrm{L})$, panobinostat (5 and $10 \mathrm{nmol} / \mathrm{L}$ ), and combination treatment of U266 for $24 \mathrm{~h}(n=3, \pm \mathrm{SE})$, (B) Percentage of active caspase-9 after treatment with Tegavivint, panobinostat, or the combination for OCIMy1 and U266 at 24 and $48 \mathrm{~h}$ respectively (raw data), Figure S4: Composite images (chemiluminescent and colorimetric)/scanned images (cyclin D2) derived from immunoblotting of OCIMy1 and U266 whole-cell lysates for $\beta$-catenin down-stream targets Myc, Cyclin D1, and Cyclin D2 after 24 or 48 h of treatment with Tegavivint, panobinostat, or their combination, Figure S5: (A) Larger view of OCIMy1 treated with DMSO for $20 \mathrm{~h}$, stained with TMRE (red for mitochondrial potential), Mito Tracker Green (green for mitochondria) and Hoechst 33342 (blue for nuclei) and evaluated with confocal microscopy, (B) OCIMy1 and U266 were treated with $100 \mathrm{nmol} / \mathrm{L}$ of Tegavivint in combination with $10 \mathrm{nmol} / \mathrm{L}$ of panobinostat or vehicle (DMSO) for $20 \mathrm{~h}$. Mitochondrial load was measured by Mitotracker Green straining and FC, Figure S6: OCIMy1 and U266 were treated with vehicle, $100 \mathrm{nmol} / \mathrm{L}$ of Tegavivint, $10 \mathrm{nmol} / \mathrm{L}$ of panobinostat, or combination for $18 \mathrm{~h}$. Analysis of ECAR was performed using Seahorse XF analyser to assess glycolysis in the context of a glycolysis stress test, Figure S7: NSG human MMbearing mice were treated with vehicle alone, $10 \mathrm{mg} / \mathrm{kg}$ of panobinostat, $30 \mathrm{mg} / \mathrm{kg}$ of Tegavivint, or the combination of panobinostat and Tegavivint, Kaplan-Meier survival curves were calculated, Figure S8: (A) Panobinostat alone, Tegavivint alone or combination treatment did not affect the GI tract of of the MM-bearing mice as validated by body weight (BW) monitoring (left panel) and IHC of colonic mucosa (right panel). (B) At completion of two treatment cycles (day 57) blood was collected and cell counts were performed. References $[37,38]$ are cited in the supplementary materials.

Author Contributions: Conceptualization, I.S., T.K. and A.S.; methodology, I.S., I.C., S.K.H., T.S., S.M. and M.d.V.; software, I.S., I.C., T.S.; validation, I.S., S.W.; formal analysis, I.S., I.C., T.S.; investigation, I.S.; resources, I.C., S.K.H., T.S., T.K., A.S. and M.d.V.; data curation, I.S.; writing-original draft preparation, I.S.; writing-review and editing, I.S., T.K., S.M. and A.S.; visualization, I.S., I.C., T.S.; supervision, T.K., A.S.; project administration, I.S., T.K.; funding acquisition, I.S., T.K., A.S. All authors have read and agreed to the published version of the manuscript.

Funding: This research received no external funding. 
Institutional Review Board Statement: Primary multiple myeloma samples were obtained from RRMM patients, following written informed consent with approval (approval code 4/05-V4, 23 September 2020) from the Alfred Hospital Research and Ethics Committee. Approval for the murine studies was obtained from the Animal Ethics Committee of the Alfred Hospital, Melbourne, Australia (E/1376/2013/M, date of approval: 16 September 2013).

Informed Consent Statement: Primary multiple myeloma samples were obtained from RRMM patients, following written informed consent with approval from the Alfred Hospital Research and Ethics Committee.

Data Availability Statement: The data presented in this study are available on request to the corresponding authors.

Acknowledgments: The authors acknowledge the Monash Histology Platform, Department of Anatomy and Developmental Biology, Monash University (Clayton, Melbourne, Australia), Monash Micro Imaging-ARA Monash University (Commercial Rd, Melbourne, Australia) and the AMREP Flow Cytometry Core Facility (Commercial Road, Melbourne, Australia). We also acknowledge the facilities and scientific and technical assistance of the National Imaging Facility, a National Collaborative Research Infrastructure Strategy (NCRIS) capability, at the Monash Biomedical Imaging, Monash University. The authors thank Iterion for kindly providing Tegavivint.

Conflicts of Interest: S.K.H. is the Chief Scientific Officer at Iterion Therapeutics. The remaining authors declare no conflict of interest.

\section{References}

1. Sung, H.; Ferlay, J.; Siegel, R.L.; Laversanne, M.; Soerjomataram, I.; Jemal, A.; Bray, F. Global cancer statistics 2020: GLOBOCAN estimates of incidence and mortality worldwide for 36 cancers in 185 countries. CA Cancer J. Clin. 2021, 71, 209-249. [CrossRef]

2. Robak, P.; Drozdz, I.; Szemraj, J.; Robak, T. Drug resistance in multiple myeloma. Cancer Treat Rev. 2018, 70, 199-208. [CrossRef] [PubMed]

3. Kumar, S.K.; Therneau, T.M.; Gertz, M.A.; Lacy, M.Q.; Dispenzieri, A.; Rajkumar, S.V.; Fonseca, R.; Witzig, T.E.; Lust, J.A.; Larson, D.R.; et al. Clinical course of patients with relapsed multiple myeloma. Mayo Clin. Proc. 2004, 79, 867-874. [CrossRef]

4. San-Miguel, J.F.; Hungria, V.T.; Yoon, S.S.; Beksac, M.; Dimopoulos, M.A.; Elghandour, A.; Jedrzejczak, W.W.; Günther, A.; Nakorn, T.N.; Siritanaratkul, N.; et al. Panobinostat plus bortezomib and dexamethasone versus placebo plus bortezomib and dexamethasone in patients with relapsed or relapsed and refractory multiple myeloma: A multicentre, randomised, double-blind phase 3 trial. Lancet Oncol. 2014, 15, 1195-1206. [CrossRef]

5. Laubach, J.P.; Schjesvold, F.; Mariz, M.; Dimopoulos, M.A.; Lech-Maranda, E.; Spicka, I.; Hungria, V.T.M.; Shelekhova, T.; Abdo, A.; Jacobasch, L.; et al. Efficacy and safety of oral panobinostat plus subcutaneous bortezomib and oral dexamethasone in patients with relapsed or relapsed and refractory multiple myeloma (PANORAMA 3): An open-label, randomised, phase 2 study. Lancet Oncol. 2021, 22, 142-154. [CrossRef]

6. Eleutherakis-Papaiakovou, E.; Kanellias, N.; Kastritis, E.; Gavriatopoulou, M.; Terpos, E.; Dimopoulos, M.A. Efficacy of panobinostat for the treatment of multiple myeloma. J. Oncol. 2020, 2020, 7131802. [CrossRef] [PubMed]

7. Anastas, J.N.; Moon, R.T. WNT signalling pathways as therapeutic targets in cancer. Nat. Rev. Cancer 2013, 13, 11-26. [CrossRef] [PubMed]

8. Spaan, I.; Raymakers, R.A.; van de Stolpe, A.; Peperzak, V. Wnt signaling in multiple myeloma: A central player in disease with therapeutic potential. J. Hematol. Oncol. 2018, 11, 67. [CrossRef]

9. Savvidou, I.; Khong, T.; Cuddihy, A.; McLean, C.; Horrigan, S.; Spencer, A. $\beta$-Catenin Inhibitor BC2059 is efficacious as monotherapy or in combination with proteasome inhibitor bortezomib in multiple myeloma. Mol. Cancer Ther. 2017, 16, 1765-1778. [CrossRef]

10. Nomura, M.; Rainusso, N.; Lee, Y.C.; Dawson, B.; Coarfa, C.; Han, R.; Larson, J.L.; Shuck, R.; Kurenbekova, L.; Yustein, J.T. Tegavivint and the $\beta$-Catenin/ALDH Axis in Chemotherapy-Resistant and Metastatic Osteosarcoma. J. Natl. Cancer Inst. 2019, 111, 1216-1227. [CrossRef]

11. Fiskus, W.; Sharma, S.; Saha, S.; Shah, B.; Devaraj, S.G.; Sun, B.; Horrigan, S.; Leveque, C.; Zu, Y.; Iyer, S.; et al. Pre-clinical efficacy of combined therapy with novel $\beta$-catenin antagonist BC2059 and histone deacetylase inhibitor against AML cells. Leukemia 2015, 29, 1267-1278. [CrossRef] [PubMed]

12. Saenz, D.T.; Fiskus, W.; Manshouri, T.; Mill, C.P.; Qian, Y.; Raina, K.; Rajapakshe, K.; Coarfa, C.; Soldi, R.; Bose, P.; et al. Targeting nuclear $\beta$-catenin as therapy for post-myeloproliferative neoplasm secondary AML. Leukemia 2019, 33, 1373-1386. [CrossRef] [PubMed]

13. Li, J.; Wng, C.Y. TBL1-TBLR1 and beta-catenin recruit each other to Wnt target-gene promoter for transcription activation and oncogenesis. Nat. Cell Biol. 2008, 10, 160-169. [CrossRef] [PubMed] 
14. Soldi, R.; Halder, T.G.; Sampson, S.; Vankayalapati, H.; Weston, A.; Thode, T.; Bhalla, K.N.; Ng, S.; Rodriguez Del Villar, R.; Drenner, K.; et al. The Small Molecule BC-2059 inhibits Wingless/integrated (Wnt)-dependent gene transcription in cancer through disruption of the Transducin $\beta$-Like 1- $\beta$-Catenin protein complex. J. Pharmacol. Exp. Ther. 2021, 378, 77-86. [CrossRef]

15. Mithraprabhu, S.; Khong, T.; Spencer, A. Overcoming inherent resistance to histone deacetylase inhibitors in multiple myeloma cells by targeting pathways integral to the actin cytoskeleton. Cell Death Dis. 2014, 5, e1134. [CrossRef]

16. Ianevski, A.; Giri, K.A.; Aittokallio, T. SynergyFinder 2.0: Visual analytics of multi-drug combination synergies. Nucleic Acids Res. 2020, 48, W488-W493. [CrossRef]

17. González-Calle, V.; Keane, N.; Braggio, E.; Fonseca, R. Precision medicine in myeloma: Challenges in defining an actionable approach. Clin. Lymphoma Myeloma Leuk. 2017, 17, 621-630. [CrossRef]

18. Tateishi, K.; Iafrate, A.J.; Ho, Q.; Curry, W.T.; Batchelor, T.T.; Flaherty, K.T.; Onozato, M.L.; Lelic, N.; Sundaram, S.; Cahill, D.P.; et al. Myc-Driven glycolysis is a therapeutic target in glioblastoma. Clin. Cancer Res. 2016, 22, 4452-4465. [CrossRef]

19. Dejure, F.R.; Eilers, M. MYC and tumour metabolism: Chicken and egg. EMBO J. 2017, 36, 3409-3420. [CrossRef]

20. Wardell, S.E.; Ilkayeva, O.R.; Wieman, H.L.; Frigo, D.E.; Rathmell, J.C.; Newgard, C.B.; McDonnell, D.P. Glucose metabolism as a target of histone deacetylase inhibitors. Mol. Endocrinol. 2009, 23, 388-401. [CrossRef]

21. Vergara, D.; Stanca, E.; Guerra, F.; Priore, P.; Gaballo, A.; Franck, J.; Simeone, P.; Trerotola, M.; De Domenico, S.; Fournier, I.; et al. $\beta$-Catenin knockdown affects mitochondrial biogenesis and lipid metabolism in breast cancer cells. Front Physiol. $2017,8,544$. [CrossRef] [PubMed]

22. Yoon, J.C.; Ng, A.; Kim, B.H.; Bianco, A.; Xavier, R.J.; Elledge, S.J. Wnt signaling regulates mitochondrial physiology and insulin sensitivity. Genes Dev. 2010, 24, 1507-1518. [CrossRef] [PubMed]

23. Gao, X.; Shen, L.; Li, X.; Liu, J. Efficacy and toxicity of histone deacetylase inhibitors in relapsed/refractory multiple myeloma: Systematic review and meta-analysis of clinical trials. Exp Ther Med. 2019, 18, 1057-1068. [CrossRef] [PubMed]

24. van Andel, H.; Kocemba, K.A.; Spaargaren, M.; Pals, S.T. Aberrant Wnt signaling in multiple myeloma: Molecular mechanisms and targeting options. Leukemia 2019, 33, 1063-1075. [CrossRef]

25. Baron, R.; Kneissel, M. WNT signaling in bone homeostasis and disease: From human mutations to treatments. Nat. Med. 2013, 19, 179-192. [CrossRef]

26. Terpos, E.; Ntanasis-Stathopoulos, I.; Dimopoulos, M.A. Myeloma bone disease: From biology findings to treatment approaches. Blood 2019, 133, 1534-1539. [CrossRef]

27. Mithraprabhu, S.; Sirdesai, S.; Chen, M.; Khong, T.; Spencer, A. Circulating tumour DNA analysis for tumour genome characterisation and monitoring disease burden in extramedullary multiple myeloma. Int. J. Mol. Sci. 2018, 19, 1858. [CrossRef]

28. Bjorklund, C.C.; Ma, W.; Wang, Z.Q.; Davis, R.E.; Kuhn, D.J.; Kornblau, S.M.; Wang, M.; Shah, J.J.; Orlowski, R.Z. Evidence of a role for activation of Wnt/beta-catenin signaling in the resistance of plasma cells to lenalidomide. J. Biol. Chem. 2011, 286, 11009-11020. [CrossRef]

29. Proto, M.C.; Fiore, D.; Piscopo, C.; Franceschelli, S.; Bizzarro, V.; Laezza, C.; Lauro, G.; Feoli, A.; Tosco, A.; Bifulco, G.; et al. Inhibition of Wnt/ $\beta$-Catenin pathway and histone acetyltransferase activity by Rimonabant: A therapeutic target for colon cancer. Sci. Rep. 2017, 7, 11678. [CrossRef]

30. Götze, S.; Coersmeyer, M.; Müller, O.; Sievers, S. Histone deacetylase inhibitors induce attenuation of Wnt signaling and TCF7L2 depletion in colorectal carcinoma cells. Int. J. Oncol. 2014, 45, 1715-1723. [CrossRef]

31. Kumar, S.K.; Harrison, S.J.; Cavo, M.; de la Rubia, J.; Popat, R.; Gasparetto, C.; Hungria, V.; Salwender, H.; Suzuki, K.; Kim, I.; et al. Venetoclax or placebo in combination with bortezomib and dexamethasone in patients with relapsed or refractory multiple myeloma (BELLINI): A randomised, double-blind, multicentre, phase 3 trial. Lancet Oncol. 2020, 21, 1630-1642. [CrossRef]

32. Gong, J.N.; Khong, T.; Segal, D.; Yao, Y.; Riffkin, C.D.; Garnier, J.M.; Khaw, S.L.; Lessene, G.; Spencer, A.; Herold, M.J.; et al. Hierarchy for targeting prosurvival BCL2 family proteins in multiple myeloma: Pivotal role of MCL1. Blood 2016, 128, 1834-1844. [CrossRef] [PubMed]

33. Luis, T.C.; Ichii, M.; Brugman, M.H.; Kincade, P.; Staal, F.J. Wnt signaling strength regulates normal haematopoiesis and its deregulation is involved in leukemia development. Leukemia 2012, 26, 414-421. [CrossRef] [PubMed]

34. Niida, A.; Hiroko, T.; Kasai, M.; Furukawa, Y.; Nakamura, Y.; Suzuki, Y.; Sugano, S.; Akiyama, T. DKK1, a negative regulator of Wnt signaling, is a target of the $\beta$-catenin/TCF pathway. Oncogene 2004, 23, 8520-8526. [CrossRef]

35. Sottnik, J.L.; Hall, C.L.; Zhang, J.; Keller, E.T. Wnt and Wnt inhibitors in bone metastasis. Bonekey Rep. 2012, 1, 101. [CrossRef]

36. Nusse, R.; Fuerer, C.; Ching, W.; Harnish, K.; Logan, C.; Zeng, A.; ten Berge, D.; Kalani, Y. Wnt signaling and stem cell control. Cold Spring Harb. Symp. Quant. Biol. 2008, 73, 59-66. [CrossRef]

37. Okugawa, H. A new imaging method for confocal microscopy. Proc. SPIE 2008, 6860, 1-7. [CrossRef]

38. Doube, M.; Kłosowski, M.M.; Arganda-Carreras, I.; Cordelières, F.P.; Dougherty, R.P.; Jackson, J.S.; Schmid, B.; Hutchinson, J.R.; Shefelbine, S.J. BoneJ: Free and extensible bone image analysis in ImageJ. Bone 2010, 47, 1076-1079. 